\title{
Electroelastic Effect in Tourmaline
}

\author{
E. Kittinger, K. Seil, and J. Tichý \\ Institute of Experimental Physics, University of Innsbruck, Austria \\ Z. Naturforsch. 34a, 1352-1354 (1979); received October 6, 1979
}

\begin{abstract}
Measuring the electric field induced frequency shift of tourmaline resonators the electroelastic constants $g_{311}$ and $\delta_{333}$ are determined. These constants may be interpreted as describing either the electric field dependence of an elastic constant or the stress (strain) dependence of a piezoelectric constant. Fields up to about $3 \times 10^{6} \mathrm{~V} / \mathrm{m}$ were used. Within this range the effect is linear and is of the same order as in $\alpha$-quartz.
\end{abstract}

\section{Introduction}

Already shortly after the discovery of the piezoelectric effect there were attempts to measure the stress or strain dependence of piezoelectric constants in quartz and tourmaline crystals [1]. These experiments failed to provide consistent results. This failure is mainly due to three reasons:

1) The effect (as has been shown later on) is small in both crystals. 2) The precision of static as well as of dynamic methods for the measurement of piezoelectric constants is not very high. 3 ) It is very difficult to induce a simple, well defined, if possible uniaxial state of stress within the specimen under investigation.

The first successful determination of the dependence of the piezoelectric modulus $e_{11}$ on the deformation $V_{1}$ is due to Graham [2] who measured the piezoelectric polarization of an $\mathrm{X}$-cut quartz plate during the passage of a shock wave propagating in the direction of the specimen thickness whereby strains from $2.4 \times 10^{-3}$ to $4.3 \times 10^{-2}$ were reached. Later Stanton and Graham $[3,4]$ performed similar experiments on $\mathrm{LiNbO}_{3}$ which shows the same crystal symmetry as tourmaline.

Thus, in fact third order material constants have been determined for which in contrast to electrostriction constants no generally accepted name has been established so far. In accordance with [5] we call them electroelastic constants. The justification for this name will follow from the next section.

\section{The Electroelastic Constants}

The electroelastic constants are defined as third order partial derivatives of the appropriate thermo-

Reprint requests to Dr. E. Kittinger, Institut für Experimentalphysik, Universität Innsbruck, Schoepfstraße 41, A-6020 Innsbruck, Österreich. dynamic potential which is differentiated once with respect to an electrical variable and twice with respect to mechanical variables. Thus the electroelastic constants are the coordinates of a fifth rank tensor. The thermodynamic potential to be used follows from the choice of independent variables, that is from the experimental conditions. Depending on whether one differentiates with respect to two mechanical variables or to one mechanical and one electrical variable a second partial derivative of a thermodynamic potential defines a second order elastic or a piezoelectric constant respectively. Consequently an electroelastic constant may be looked upon as describing the dependence either of an elastic constant on an electrical quantity or of a piezoelectric constant on a mechanical quantity.

When defining second order material constants, the difference in energy density as referred to unit volume of the strained body and to unit volume of the unstrained body may be neglected. For third order constants this approximation is no longer justified. Accordingly in nonlinear theories the coordinates $V_{\lambda}$ of the Lagrangian finite strain tensor are used to describe the deformation and the coordinates $T_{\lambda}$ of the so called thermodynamic stress tensor to characterize the state of stress. For reviews of this topic see [6] and [7]. In the same sense the electric variables (in this case the electric field $E_{i}$ ) are to be referred to the unstrained reference state. Quite generally speaking, one has to use so called "reduced densities" of the thermodynamic potentials in order to define the variables of state [8]. In experimental determinations of these third order constants (where usually global quantities, such as forces, electric charges and voltages are measured) such an interpretation of the pertinent quantities usually is tacitly implied. 
In agreement with usual experimental conditions we choose as the thermodynamic potential corresponding to the adiabatic case the enthalpy $H$ (for the isothermal case, that is for quasistatic measurements, it would be the Gibbs potential) and we can write

$g_{i \lambda \mu}=-\left(\frac{\partial^{3} H}{\partial E_{i} \partial T_{\lambda} \partial T_{\mu}}\right)_{0}=\left(\frac{\partial s_{\lambda \mu}}{\partial E_{i}}\right)_{0}=\left(\frac{\partial d_{i \lambda}}{\partial T_{\mu}}\right)_{0}$

With $g_{i \lambda \mu}$ we denote the electroelastic coefficients, $s_{\lambda \mu}$ are second order elastic coefficients and $d_{i \lambda}$ are piezoelectric coefficients.

Matrix notation in the sense of Brugger [9] is used throughout. The subscript 0 indicates that the quantities enclosed in the brackets are the values of the functions for $E_{i}=0, T_{\lambda}=0$.

In addition we define the electroelastic moduli $\delta_{i \lambda \mu}$ with the aid of the "electric enthalpy" $\tilde{H}$ by the relation

$\delta_{i \lambda \mu}=-\left(\frac{\partial^{3} \tilde{H}}{\partial E_{i}} \frac{\partial V_{\lambda} \partial V_{\mu}}{{ }^{\prime}}=\left(\frac{\partial c_{\lambda \mu}}{\partial E_{i}}\right)_{0}=-\left(\frac{\partial e_{i \lambda}}{\partial V_{\mu}}\right)_{0}\right.$,

where $c_{\lambda \mu}$ denote the elastic moduli and $e_{i \lambda}$ the piezoelectric moduli.

\section{Experiment}

Equations (1) and (2) indicate two different ways of determining the respective electroelastic constants experimentally. The first of these is represented by the method of Graham [2,3], which has already been mentioned; the second is based on the measurement of field induced changes of elastic constants. This may be accomplished for instance via the resonance frequency shift which is observed on application of a DC electric field to a piezoelectric resonator. This second principle of measurement was developed by Hruška [10] and was used later also by others $[11,12]$.

The major advantages of this method are a comparatively simple experimental arrangement and the high precision possible in measuring the resonance frequency shift. Making use of this method we have measured the constants $g_{311}$ and $\delta_{333}$ for tourmaline using specimens in the form of bars and discs.

The fundamental resonance frequency for length extensional vibrations of a bar of orientation $\mathrm{ZX}$ $[13]$ is known to be

$$
f=\frac{1}{2 l} \sqrt{\frac{1}{\varrho s_{11}}},
$$

where $l$ denotes the length, $\varrho$ the density and $s_{11}$ the elastic coefficient of the bar. An electric field $E_{3}$ applied via electrodes which are perpendicular to the $x_{3}=Z$-axis brings about a change in resonance frequency which follows from (3) as

$$
\frac{1}{f}\left(\frac{\partial f}{\partial E_{3}}\right)_{0}=\frac{1}{2}\left\{d_{33}-\frac{1}{s_{11}}\left(\frac{\partial s_{11}}{\partial E_{3}}\right)_{0}\right\} .
$$

The first term in the brackets represents the contribution of the reciprocal piezoelectric effect which changes $l$ as well as $\varrho$. The second term gives the electroelastic coefficient to be determined.

\section{Results}

Experimental data of $\Delta f$ vs. $E_{3}$ are shown in Figure 1. Measurements were performed at room temperature with bars having resonance frequencies of the order $10^{5} \mathrm{~Hz}$. For symmetry reasons $g_{311}$ equals $g_{322}$ for tourmaline. Thus our measurements yield the result

$$
\begin{aligned}
g_{322} & =g_{311}=\left(\partial s_{11} / \partial E_{3}\right)_{0}=\left(\partial d_{31} / \partial T_{1}\right)_{0} \\
& =-2.59 \times 10^{-23} \mathrm{~m}^{3} \mathrm{~N}^{-1} \mathrm{~V}^{-1}
\end{aligned}
$$

Analogously, the electroelastic modulus $\delta_{333}$ was determined at room temperature from thickness longitudinal vibrations of $\mathrm{Z}$-cut circular dises (resonance frequency about $2 \mathrm{MHz}$ ):

$$
\begin{aligned}
\delta_{333} & =\left(\partial c_{33} / \partial E_{3}\right)_{0}=-\left(\partial e_{33} / \partial V_{3}\right)_{0} \\
& =2.22 \mathrm{Nm}^{-1} \mathrm{~V}^{-1} .
\end{aligned}
$$

Our values of the electroelastic constants are referred to a coordinate system as introduced by Cady [1]. The piezoelectric and elastic constants

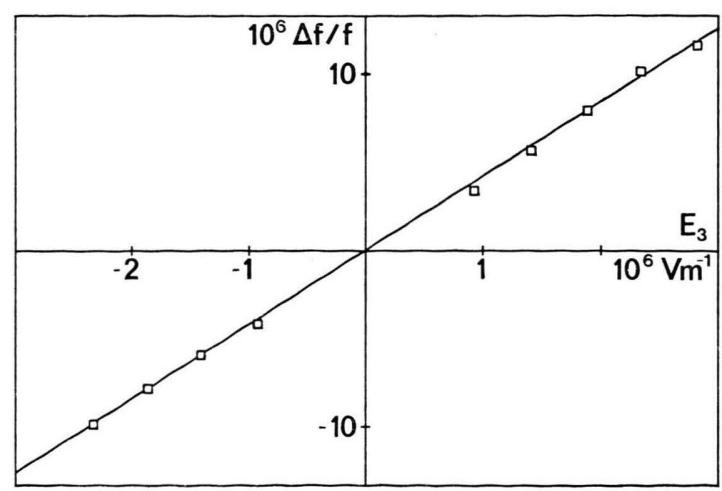

Fig. 1. Relative resonance frequency shift of a bar resonator as a function of the applied DC electric field. 
used in (4) and in the corresponding equation for $\delta_{333}$ were taken from Mason [14] after adjustment of the signs to this coordinate system.

From Fig. 1 it is seen that within experimental accuracy the dependence of resonance frequency on the electric field is linear and does not depend on the polarity of the field. In this respect tourmaline is similar to quartz, while in ammonium dihydrogen phosphate (ADP) the dependence, within the same range of field strength, also contains a noticeable quadratic effect $[11,15]$.

Results for different specimens agreed within $10 \%$. As far as accuracy is concerned one should bear in mind that tourmaline occurs in several modifications varying in chemical composition which accordingly show considerable scatter in lattice parameters and density. To our knowledge there exists no detailed investigation of piezoelectric properties of the different modifications [16]. With the exception of the hydrostatic piezoelectric

[1] W. G. Cady, Piezoelectricity, Dover Publications Inc., New York 1964

[2] R. A. Graham, Phys. Rev. B 6, 4779 (1972).

[3] P. L. Stanton and R. A. Graham, Applied Physics Letters 31, 723 (1977).

[4] R. A. Graham, J. Appl. Phys. 48, 2153 (1977).

[5] K. Hruška, IEEE Transactions on Sonics and Ultrasonics SU-18, 1 (1971).

[6] R. N. Thurston, Wave Propagation in Fluids and Normal Solids, in Physical Acoustics, ed. W. P. Mason, Academic Press, New York 1964.

[7] R. N. Thurston, Waves in Solids, in Handbuch der Physik, ed. S. Flügge, Vol. VIa/4, Springer, Berlin 1974.

[8] P. Hájiček, Czech. J. Phys. B 17, 613; 969 (1967); B 18, 1008 (1968); B 19, 26 (1969).

[9] K. Brugger, Phys. Rev. 133, A 1611 (1964).

[10] K. Hruška, Czech. J. Phys. B 11, 150 (1961); B 12, 338 (1962). coefficient [17] there appear to exist no newer values than those given by Mason [14]. Taking this into account, our results should be taken as a first and preliminary investigation of the electroelastic effect in tourmaline. On account of the symmetry (class $3 \mathrm{~m}$ ), for a complete description of the effect it would be necessary to determine 13 independent coordinates of the electroelastic tensor.

Aside from the purely physical interest an investigation of the electroelastic effect in tourmaline has some practical consequences as nowadays quartz elements in piezoelectric pressure gauges and accelerometers for high temperature applications (above approximately $300{ }^{\circ} \mathrm{C}$ ) are frequently replaced by tourmaline. In such applications the linear dependence of the piezoelectric polarization on stress or deformation is tacitly presumed. Our results show that for Z-cut tourmaline, which is most widely used, this assumption holds about as well as for an $\mathrm{X}$-cut quartz plate [18].

[11] K. Seil, Thesis, University of Innsbruck 1978

[12] A. Kinigadner, Thesis, University of Innsbruck 1978.

[13] IRE Standards on Piezoclectric Crystals, Proc. IRE 37, 1378 (1949)

[14] W. P. Mason, Piezoelectric Crystals and Their Application to Ultrasonics, D. van Nostrand, New York 1950 .

[15] K. Hruška, IEEE Transactions on Sonics and Ultrasonics SU.24, 54 (1977).

[16] W. R. Cook and H. Jaffe, Piezoelectric, Electrostrictive and Dielectric Constants, and Electromechanical Coupling Factors of Diezoelectric Crystals, in: Landolt-Börnstein, New Series, ed. K.-H. Hellwege, Vol. III/11, Springer-Verlag, Berlin 1979.

[17] I. S. Zheludev and M. M. Tagieva, Soviet Phys.Cryst. 7, 473 (1963).

[18] K. Hruška, Proceedings of the 31st Annual Frequency Control Symposium, Atlantic City, New Jersey 1977. 